\title{
Zbtb20 modulates the sequential generation of neuronal layers in developing cortex
}

\author{
Anton B. Tonchev ${ }^{1,2,3^{*}}$, Tran Cong Tuoc ${ }^{2,4}$, Eva H. Rosenthal ${ }^{1}$, Michèle Studer ${ }^{5,6}$ and Anastassia Stoykova ${ }^{1,2,3^{*}}$
}

\begin{abstract}
Background: During corticogenesis, genetic programs encoded in progenitor cells at different developmental stages and inherited in postmitotic neurons specify distinct layer and area identities. Transcription factor Zbtb20 has been shown to play a role for hippocampal development but whether it is implicated in mammalian neocortical morphogenesis remains unknown.

Results: Here, we report that during embyogenesis transcription factor Zbtb20 has a dynamic spatio-temporal expression pattern in mitotic cortical progenitors through which it modulates the sequential generation of cortical neuronal layer identities. Zbtb20 knock out mice exhibited enhanced populations of early born L6-L4 neuronal subtypes and a dramatic reduction of the late born L3/L2 neurons. This defect was due to a temporal misbalance in the production of earlier versus later born neurons, leading to a progressive diminishing of the progenitor pool for the generation of L3-L2 neurons. Zbtb20 implements these temporal effects in part by binding to promoter of the orphan nuclear receptor CoupTF1/Nr2f1. In addition to its effects exerted in cortical progenitors, the postmitotic expression of Zbtb20 in L3/L2 neurons starting at birth may contribute to their proper differentiation and migration.
\end{abstract}

Conclusions: Our findings reveal Zbtb20 as a novel temporal regulator for the generation of layer-specific neuronal identities.

Keywords: Zbtb20, Development, Neocortex, Temporal identity, Transcription factor

\section{Background}

The mammalian neocortex (Ncx), in which neurons are arranged radially in six layers and tangentially in numerous functional domains, is a recent acquisition in brain evolution. During development, the majority of cortical glutamatergic neurons are generated by radial glial cells (RGCs) in the germinative ventricular (VZ) and subventricular (SVZ) zone of the dorsolateral pallium. Generation of neuronal sets with a layer-specific identity depends on an intrinsically encoded genetic program and environmental cues acting during the S-phase of the mitotic cycle [1]. Neurons with different fates are produced according to an "inside-first outside-last" schedule: first, lower layer (LL) neurons (L6/L5), followed by generation of the upper layer (UL) neurons (L4/L3/L2).

\footnotetext{
* Correspondence: anton.tonchev@mu-varna.bg; astoyko@gwdg.de

${ }^{1}$ Molecular Developmental Neurobiology Laboratory, Max Planck Institute of

Biophysical Chemistry, Am Fassberg, 37077 Gottingen, Germany

Full list of author information is available at the end of the article
}

During mouse development, the layer specific neuronal subtypes are generated throughout embryonic (E) stages E10.5 - E17.5 in partially overlapping time windows with a peak for generation at E11.5 for L1, E12.5 (L6), E13.5 (L5), E14.5 (L4) and E16.5-E17.5 (L3-L2) [2-5]. The birthdate of cortical neurons is directly also related to their projection identity. Thus, while the early born L6 and L5 neurons extend outside the brain thalamocortical (TCA) and corticospinal motor neuron (CSMN) projections, the late-born UL neurons make interhemispheric (callosal) projections inside the brain [6]. Increasing recent evidence support the view that the precise temporal programs for production of LL and UL neuronal fates relies on intrinsic mechanisms in early and late progenitors, respectively, characterized by specific combinatorial expression of TFs at distinct developmental time points [7-13]. For instance, suppression of the expression of Foxg1 at E10.5 is required to make a switch from generation of reelin-positive Cajal-Retzius 
cells, located in the marginal zone (MZ) of the cortex, to the production of neuronal subsets located in cortical plate (CP) [14]. Furthermore, while Fezf2 and Otx1 expression in apical $\mathrm{VZ}$ progenitors controls the fate specification of the LL neurons $[15,16]$, the expression of Svet1, Cux1 and Cux2 during later stages of neurogenesis in SVZ progenitors seems to specify UL neuronal fate [17-20]. The expression of TFs in postmitotic CP neurons may regulate through feedback signalling mechanism the progenitor progeny in the germinative zone [21] or the fate of the postmitotic neurons [7-13].

Neurons with distinct morphology, connectivity, neurotransmitter usage and function are tangentially organized in numerous functional domains, implicating that mechanisms of layer and area formation are interrelated. According to the current view, cortical arealization is presaged by encoded positional information ("protomap") through graded expression of sets of TFs along the anteroposterior and mediolateral axis in the two germinative zones of the neocortex $[5,22]$. Disruption of the graded expression of such TFs in VZ/SVZ leads to severe defects in the areal size and location in the Ncx [23-25]. As recently shown, affecting the intrinsic genetic mechanisms encoded by the graded expression of TF Pax6 in cortical progenitors results in an altered size of cortical somatosensory (SS) area and in parallel alterations in the sensory thalamus involving selective death of neurons in particular thalamic nuclei. Consequently, a new type of "top-down plasticity" driven by competition-mediated axon elimination and neuronal apoptosis re-patterns the sensory thalamus [26].

In a microarray screen aimed to find out genes with graded expression in the developing cortex, we identified TF $Z$ btb20 as a gene showing caudal-high to rostral-low expression gradient in VZ of E16.5 cortex, and subsequently maintains a restricted high expression in the adult hippocampus (Hi) [27]. The gene Zbtb20 (also named DPZF [28], HOF [29] or ZNF288) encodes a TF, belonging to the POK-family of BTB zinc finger transcriptional repressors, implicated in developmental processes and cancer [30]. Zbtb20 has been localized exclusively in immature post-mitotic neurons in $\mathrm{Hi}$ and migrating granule cell precursors of DG [29]. Previous research, using both gain-of-function (GOF) [31-33] and loss-of-function (LOF) [34, 35] approaches, has described the important role of TF Zbtb20 in specifying the medial pallium, the anlage of the Hi formation.

Recent studies have implicated Zbtb20 mutations in human neurodevelopmental syndromes associated with behavioral abnormalities, including intellectual disability [36], Primrose syndrome [37], autism [38], and schizophrenia [39]. The reported alterations in brain morphology in these disorders suggest possible cortical involvement beyond the hippocampus. However, no data are presently available on the involvement of Zbtb20 in neocortical morphogenesis.
In this study, we present first evidence that TF $Z b t b 20$ exerts a dynamic expression in the germinative zones of the cortex (pallium), marks specifically the uppermost L3-L2 cortical neurons and exerts a crucial control in the timely generation of distinct neuronal fates throughout cortical neurogenesis.

\section{Results}

\section{Dynamic Zbtb20 expression in telencephalic progenitors}

By E11.5 a gradient of Zbtb20-lacZ activity was evident in the $\mathrm{VZ}$ of the lateral pallium (LP) of the heterozygous Zbtb20 ${ }^{\text {lacZ/+ }}$ embryos (Additional file 1: Figure S1A, arrow). Between E12.5 and E13.5, Zbtb20 immunostaining confirmed a strong signal in both the ventral (VP) and lateral (LP) pallium (Fig. 1a, arrow). Double staining for Zbtb20 and the pallial progenitor marker Pax6 revealed a nearly complete co-expression at E13.5 (Fig. 1b1-b6) in LP (101 out of 101 assayed Zbtb20 ${ }^{+}$cells co-expressed Pax6 (100\%, $n=3)$. In VP, however, only $40 \%$ of the Zbtb20 ${ }^{+}$cells co-expressed Pax6 (59 out of

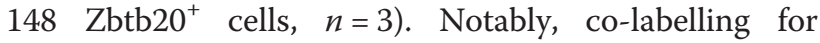
Zbtb20 and TF CoupTF1, which at this stage is expressed by both pallial and subpallial progenitors, showed a complete co-expression in LP and VP (143 out of 143 Zbtb20+ cells in LP/VP $(n=3)$ co-expressed CoupTF1 (Fig. 1c1-c6).

By E14.5-E15.5 the expression of Zbtb20 was spread throughout the entire pallial VZ (Fig. 1d; Additional file 1: Figure S1B, arrows). Co-staining for Zbtb20, RGC marker Pax6 and the mitotic marker phosphorylated vimentin (pVim) confirmed that TF Zbtb20 is expressed in dividing RGCs at the apical surface of VZ (Fig. 1e1e4, arrows; 90 out of 93 assayed pVim ${ }^{+}$cells on the apical surface were co-labeled for Zbtb20, $97 \%, n=3$ ).

At early postnatal (P) stages (P4), Zbtb20 immunosignal was evident in the most superficial layers of LP/ DP (Fig. 1f, arrow), where Zbtb20 $0^{+}$cells co-expressed Satb2 (Fig. 1g1-g3, arrows; 102 out of 129 assayed

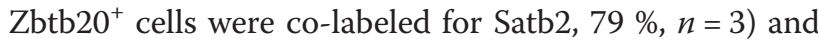
Brn2 (Additional file 2: Figure S2A1-A3, arrows; 111 out of 121 assayed Zbtb20 ${ }^{+}$cells were co-labeled for Brn2, $93 \%, n=3)$, markers of UL neocortical neurons [40, 41]. However, Zbtb20 did not co-localize with neither the L5 marker Ctip2 (Additional file 2: Figure S2B1-B3) nor the L4 marker ROR [42] (Additional file 2: Figure S2C1-C3), suggesting that the Zbtb20 expression in UL neurons is restricted to L2-L3 neurons. The expression pattern in ULs was preserved at P8 but almost disappeared at P12 (data not shown). In the early postnatal SVZ, Zbtb20 also maintained a strong expression in Nestin + RGCs (Fig. 1h1-h3; 90 out of 92 assayed Zbtb20 ${ }^{+}$cells in SVZ were co-labeled for Nestin, $92 \%, n=3$ ), suggesting a possible involvement in postnatal neurogenesis. 

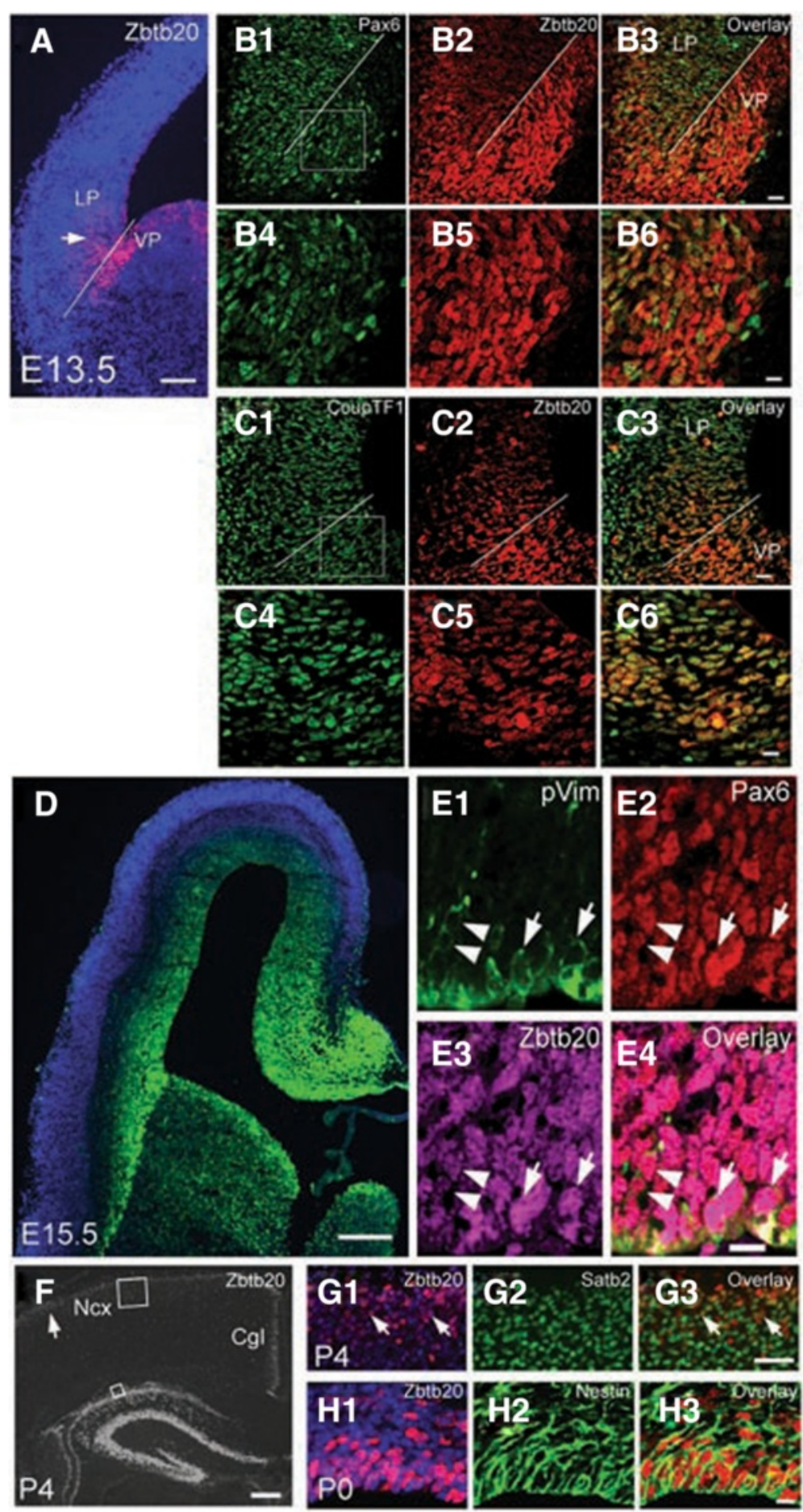

Fig. 1 (See legend on next page.) 
(See figure on previous page.)

Fig. 1 Expression of TF Zbtb20 in the developing pallium. a Immunostaining for Zbtb20 at E13.5 demarcates a strong signal just ventral to the cortico-striatal sulcus (white line) in VP and a weaker gradient in LP (arrow) gradually disappearing toward DP. b1-b6 Double-labelling for Pax6 (b1/b4) and Zbtb20 (b2/b5), and an overlay (b3/b6) in VZ of LP and VP at E13.5. c1-c6 Double-labelling for CoupTF1 (c1/c4) and Zbtb20 (c2/c5), and an overlay (c3/c6) in VZ of LP and VP at E13.5. The cortico-striatal sulcus is depicted by a white line. $\mathbf{d}$ Zbtb20 IHC on a cross brain section at stage E15.5 demonstrates strong immunosignal in germinative zones of both pallium and subpallim. e1-e4 Triple IHC with antibodies for phospho-vimentin (pVim, E1), Pax6 (e2) and Zbtb20 (e3), and an overlay (e4) in E16.5 DP. Arrows depict triple-positive cells at the apical surface, arrowheads depict a more basally located dividing cell. $\mathbf{f} \Perp \mathrm{HC}$ for Zbtb20 on coronal WT brain sections at P4. The arrow points to the Zbtb20 expression in the uppermost neocortical layers. The frames depict the position of the micrographs shown in G1-G3. g1-g3 Double immunostaining for Zbtb20 (g1, counterstained by DAPI) and Satb2 (g2), and an overlay (g3) on P4 brain cross sections. Arrows depict double-positive cells in the uppermost layers. h1-h3 Double immunostaining for Zbtb20 ( $\mathrm{H} 1$, counterstained by DAPI) and Nestin (h2), and an overlay (h3) on P0 brain cross sections depicts almost complete co-staining in VZ/SVZ. Cgl, cingulate cortex; DP, dorsal pallium; LP, lateral pallium; Ncx, neocortex; VP, ventral

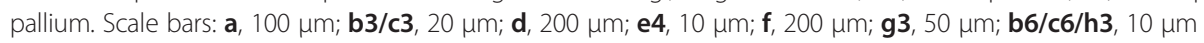

In summary, beginning at E11.5 in VZ of VP/LP, the expression of TF Zbtb20 expands into the VZ of the entire pallium at E14.5 and thereafter, suggesting that the timed expression of Zbtb20 may be involved in generation and/or specification of the UL neurons.

\section{Disturbances in superficial neocortical layers in Zbtb20 loss-of-function}

Cresyl violet (Nissl) staining of P10 coronal brain sections revealed apparent defects in cortical layering in Zbtb20 lacZ/lacZ mice (Fig. 2a1-b2). While the LLs (L6, L5) appeared overrepresented in the Zbtb20 ${ }^{\text {lacZ/lacZ }}$ somatosensory (SS) cortex, the ULs (L4-L2) seemed thinner (Fig. 2b1-b2). This impression was confirmed by NeuN immunoassaying (Fig. 2b3-b4). In order to investigate the UL disturbances in more detail, we performed immunostaining for specific UL neuronal markers. Given the widespread expression of Zbtb20 in VZ of the entire pallium at E14.5, we first investigated the expression of the L4 marker ROR [43]. Intriguingly, in the mutant cortex, the thickness of L4 was greatly augmented (Fig. 2c1c3). This notion was confirmed by another marker with a strong expression in L4, TF CoupTF1 (see below). Immunoassaying for the global (L2-L4) UL marker Cux1 $[18,19]$, however, showed a reduction of UL neurons (Fig. 2d1-d3). Additional UL markers, Brn2 (L2, L3, and L5; [40, 41]) and Satb2 (L4-L2; [7, 8]) confirmed the strongly diminished representation of UL subsets in the Zbtb20 lacZ/lacZ mice (Fig. 2h1-i3).

The overall reduction of ULs, accompanied by the selective expansion of L4, raised a question on the status of the L2-L3 neurons in the mutant cortex. Therefore, we quantified the L3-L2 $\mathrm{Cux}^{+} / \mathrm{ROR}^{-}$population (Fig. 2f2, g2, arrow), which was markedly depleted (Fig. 2g3), in contrast to the $\mathrm{L} 4 \mathrm{Cux}^{+} / \mathrm{ROR}^{+}$population, which was augmented (Fig. 2g4). In order to study whether L3 or L2 neuronal subsets were specifically affected, we made use of the expression patterns of TF FoxP1, which is expressed in L6a and L5-L3 [44] and TF Brn2, which specifically marks L3 and L2 [40, 41]. Double immunostaining for FoxP1 and Brn2 revealed an ectopic expansion of FoxP1 expression into the normal position of L2 in the mutant cortex, while cells specifically fated to L2 identity (Brn2 ${ }^{+} / \mathrm{FoxP}^{-}$, Fig. 2j1-j2, arrows) were almost completely missing (Fig. 2j3). The depletion of the ULs was not due to an enhanced apoptosis, as studied by the expression of activated Caspase-3 (data not shown). To investigate whether the molecular boundary between L5 and L4 was preserved, we applied Ctip2 (L5)/ROR (L4) double immunohistochemical (IHC) staining, and we found that these two subpopulations of cortical neurons were properly segregated (Fig. 2k1-k2).

To sum up, these findings indicate that Zbtb20 deficiency results in a significant diminishing of L3 and especially L2 neuronal subsets as well as in an augmented and ectopic presence of L4 neurons in UL position.

\section{Enhanced deep layers and normal arealization in Zbtb20 lacz/lacz cortex}

To study quantitatively the apparent enhancement of both L5 and L6 sets in the mutants (Fig. 2b1-b4), we performed IHC staining and counting of cells in the SS cortex on cross sections of both genotypes with antibodies for TFs FoxP2 [44] in L6 (Fig. 3a1-a4), Tbr1 [9] in L6 (Fig. 3b1-b4) and Ctip2 [15, 45] in L5 (Fig. 3c1c4). Indeed, the results revealed a statistically significant increased number of both L6 and L5 neurons in the mutant as compared with the control Ncx (Fig. 3a5, b5, c5). Furthermore, ISH staining of sagittal P4 brain sections indicated that the mutant cortex displayed enhanced LL neuronal subsets, including Fezf2 ${ }^{+}$L5 [15, 46] (Additional file 3: Figure S3A1-A4), Clim1 ${ }^{+}$L5 [46] (Additional file 3: Figure S3B1-B4), and $I d 2^{+} \mathrm{L} 5$ and $\mathrm{Id}^{+}$L6 [47] (Additional file 3: Figure S3C1-C4). In order to confirm that the described layering abnormalities in the $Z b t b 20^{\text {lacZ/lacZ }}$ mice are not restricted to the SS cortex, we studied the patterning of the primary motor cortex and, similarly to SS cortex, we found a decrease of the ULs and enhancement of the LLs (Additional file 4: Figure S4). 


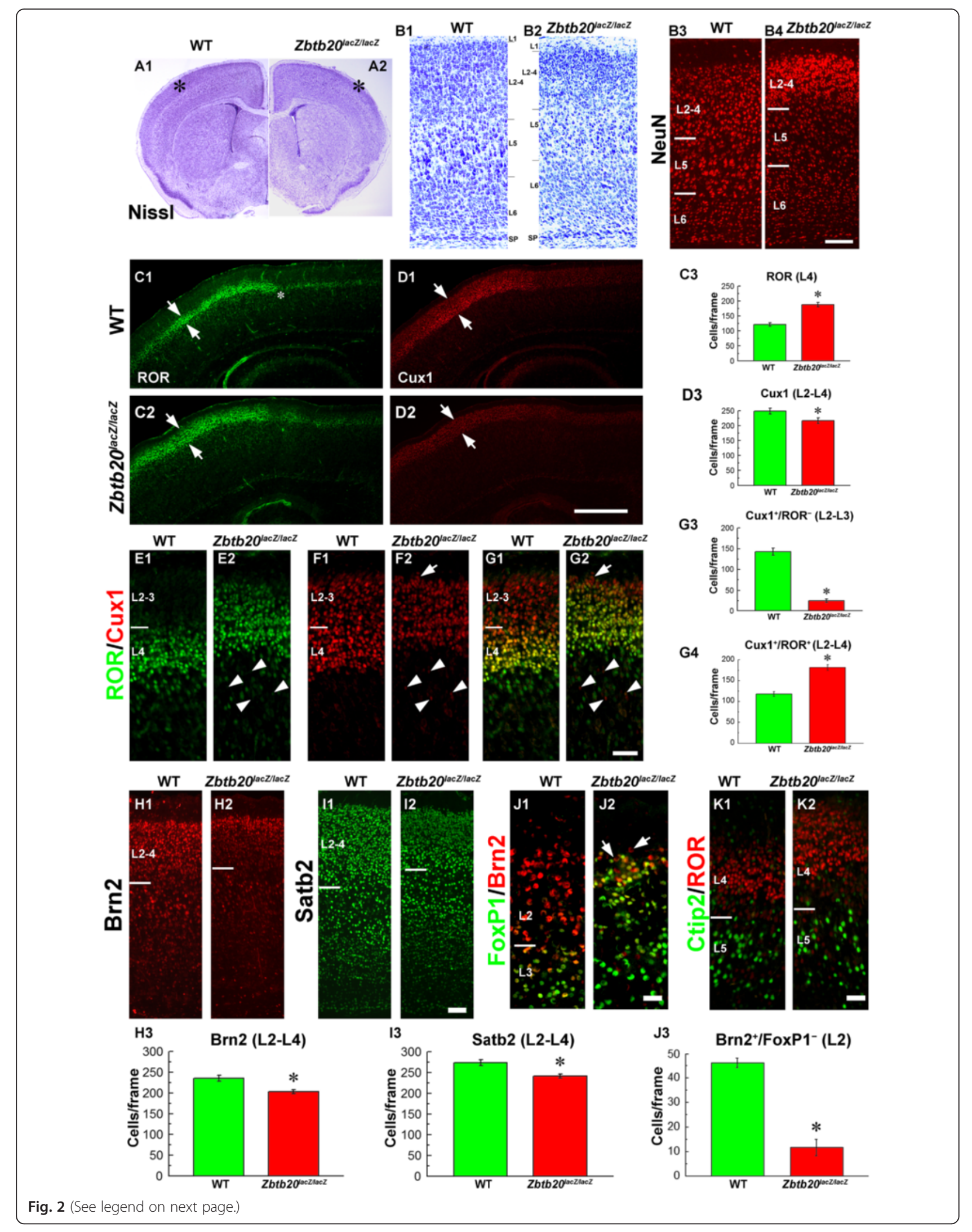


(See figure on previous page.)

Fig. 2 Upper layer defects in Zbtb2 daczlacz mice. a1-b2 Cresyl violet staining of brain cross sections from P28 wild type (WT) and mutant cortex. $(\mathrm{b} 1, \mathrm{~b} 2)$ are images at high magnification from fields in the somatosensory cortex, labeled with * in A1/A2. (b3-b4) NeuN IHC at stage P4. c1-g4 Evaluation of UL neuronal fate by using ROR and Cux1 antibodies as markers, and a statistical analysis. In (c1-c2) note the expansion of the ROR signal (arrows), while in (d1-d2), a slight reduction of the Cux1-positive band (arrows) in the mutant Ncx. (e1-g2) are higher magnifications of SS cortex, labeled with the same markers. (c3,d3,g3,g4) represent graphs of statistical analysis of the number of positive cells/frame in SS cortex ( ${ }^{*}, P<0.05, n=3$ per genotype). h1-i2 IHC with Brn2 and Satb2 antibodies as UL markers on cross sections from P4 brains, and a statistical analysis of the number of stained cells. Note the reduction of the ULs in the mutant, which was statistically confirmed $(*, P<0.05, n=3$ per genotype). j1-j3 Double IHC with Brn2 and FoxP1 antibody to evaluate UL neurons with L2 identity (Brn2 ${ }^{+} / F o x P 1^{-}$). Note the drastic diminishing of the number of L2 neurons in the mutant Ncx (arrows), which is supported by a statistical analysis ( ${ }^{*}, P<0.05, n=3$ per genotype). k1-k2 Double immunostaining for ROR (L4) and Ctip2 (L5) reveals a preserved molecular border between the two layers in the mutant. Countings of positive cells were performed in frames sized $300 \mu \mathrm{m}(\mathrm{h}) \times 100 \mu \mathrm{m}(\mathrm{w})$ spanning the ULS of SS cortex. Scale bars: b4, $100 \mu \mathrm{m} ; \mathbf{d} \mathbf{2}, 500 \mu \mathrm{m} ; \mathbf{g} \mathbf{2} / \mathbf{j} \mathbf{2}, 50 \mu \mathrm{m} ; \mathbf{i} \mathbf{2} \mathbf{k} \mathbf{2}, 100 \mu \mathrm{m}$

In layer 5 , the rostral limit of expression of $\mathrm{TF} I d 2$ outlines the position of the border between the motor/ somatosensory (M/SS) cortex, which is also marked by the caudal limit of Id2 expression in L3-L2 [48]. In $Z b t b 20^{\text {lacZ/lacZ }}$ cortex, the M/SS boundary appeared rostrally displaced into the $\mathrm{M}$ field (Additional file 3: Figure S3C1-C2, asterisk). We therefore examined other cortical area-specific markers, including Cadherin-8 (Additional file 3: Figure S3D1-D2, asterisk), Serotonin (Additional file 3: Figure S3E1-E2, asterisk), Bhlhb5 (Additional file 3: Figure S3 F1-F2, asterisk), ROR (Fig. 2c1-c2, asterisk, and data not shown), Cadherin-6 and Lmo3 (data not shown). None of these markers exhibited shifts in the mutants along the antero-posterior axis, so we concluded that the neocortical arealization in
Zbtb20 lacZ/lacZ mice is grossly not affected. This notion is also supported by the preserved pattern of the graded expression of TFs Pax6, Emx2, Foxg1 and Lhx2 in Zbtb20 lacZ/lacZ embryonic pallium at E12.5 [35], a stage at which these TFs are known to have crucial roles in specification of the intrinsic program of cortical arealization encoded in the progenitors [23].

\section{ZBTB20 modulates the temporal onset for generation of distinct neuronal layer identities}

To investigate whether Zbtb20 controls the switch to generation of neurons with different layer identities, we performed BrdU birthdating experiments at E12.5, E14.5 and E16.5 when predominantly LLs (L6, L5), L4 or L3-L2 neurons are born, respectively (Fig. 4). Taking

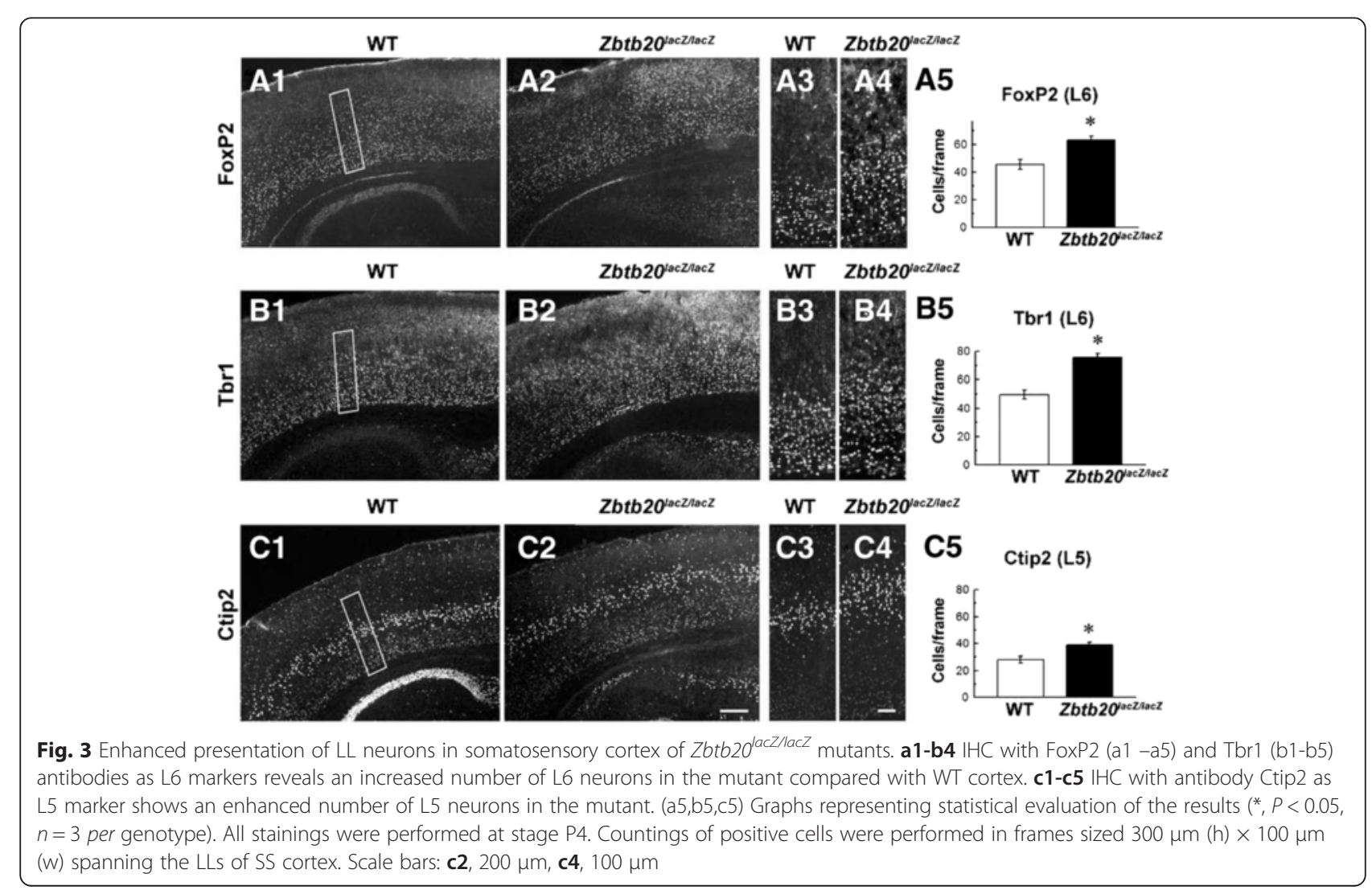




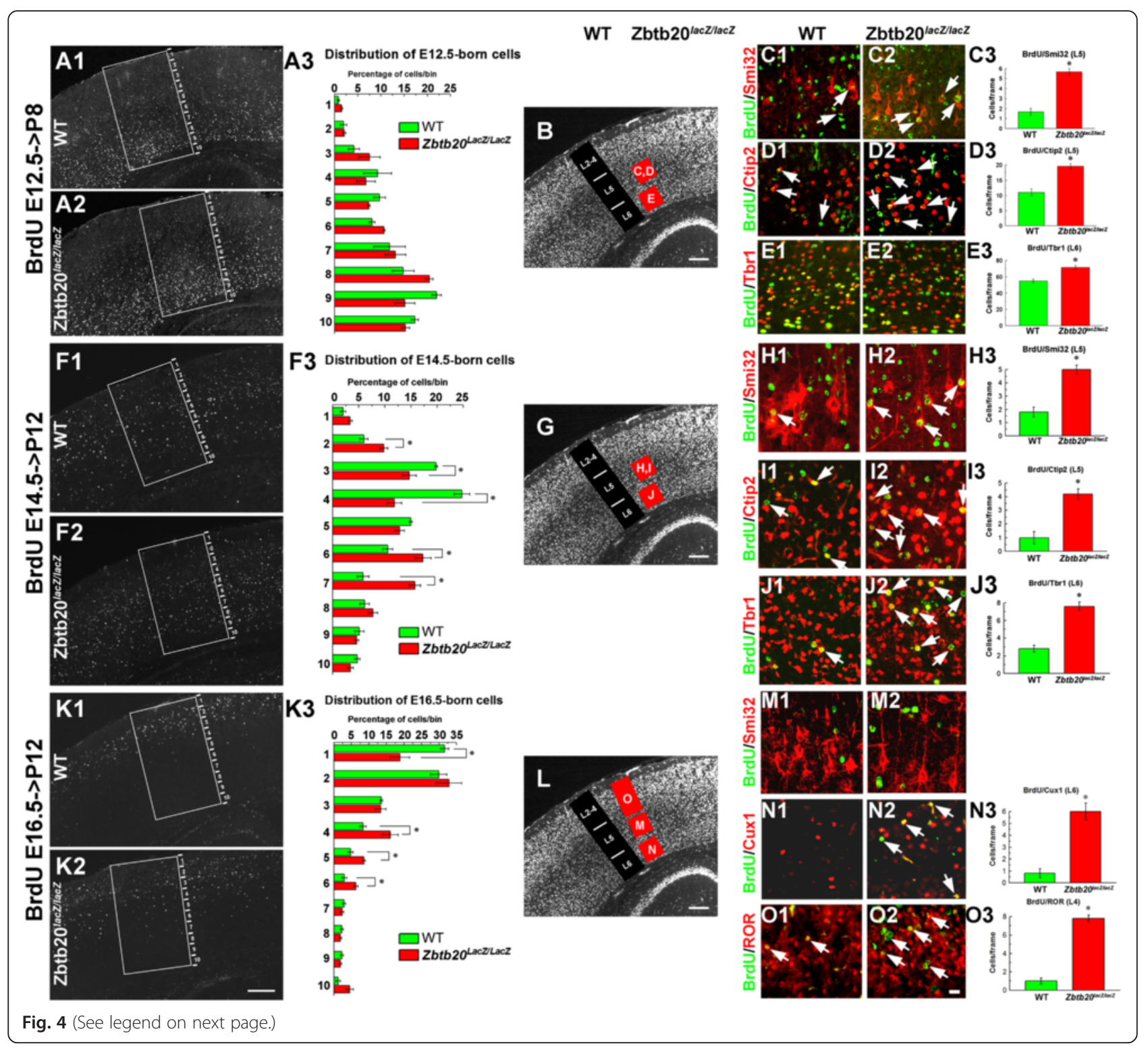


(See figure on previous page.)

Fig. 4 Laminar distribution and fate of neurons born at E12.5, E14.5 and E16.5 in WT and Zbtb20laczllacz neocortex. BrdU was injected at each of the above indicated embryonic stages and the distribution of the BrdU-tagged cells was evaluated in the postnatal SS cortex. a1-e3 Distribution and fate of cells born at E12.5 and investigated at P8. (a1-a3) Overview images of the localization of BrdU ${ }^{+}$cells to bins 1-10 and a statistical analysis showing no significant differences between WT and mutant in any of the bins. b NeuN IHC providing an overview of the cortical layers and the position of frames where counting was done in c1-e3 (red squares with respective letters). (c1-e2) Cell fate of E12.5-generated neurons in L5 (co-stained for Neurofilament-H/Smi32 and Ctip2) and L6 (co-labeled for Tbr1), and a statistical analysis $\left(\mathrm{c} 3, \mathrm{~d} 3, \mathrm{e} 3,{ }^{*}, P<0.05, n=3\right.$ per genotype). Double-labeled cells are depicted by arrows. $\mathbf{f} 1-\mathbf{j} 3$ Distribution and fate of cells born at E14.5 and investigated at P12. (f1-f3) Overview images of the localization of BrdU ${ }^{+}$cells to bins 1-10 and a statistical analysis showing a significant increase of the proportion of $\mathrm{BrdU}^{+}$cells distributed in the lower bins (6-7), and a reduction of cells localized in upper bins (3-4) in the mutant cortex as compared to WT. (g) NeuN IHC providing an overview of the cortical layers and the position of frames where counting was done in h1-j2 (red squares with respective letters). (h1-j3) Fate of E14.5-generated neurons with L5 identity (co-stained for Neurofilament-H/ Smi32 and Ctip2) and L6 (co-labeled for Tbr1), and a statistical analysis ( $\mathrm{H} 3,13, \mathrm{J3},{ }^{*}, P<0.05, n=3$ per genotype). Double-labeled cells are depicted by arrows. k1-o3 Distribution and fate of cells born on E16.5 in P12 SS cortex. (k1-k3) Overview images of the localization of BrdU ${ }^{+}$ cells to bins 1-10 and a statistical analysis showing a significant increase of the proportion of $\mathrm{BrdU}^{+}$cells localized in bins 4-6 in mutant, while a reduction of the percentage of cells localized in uppermost position in bin 1 as compared to WT. (I) NeuN IHC providing an overview of the cortical layers and the position of frames where counting was done in m1-o2 (red squares with respective letters). (m1-o3) Fate of E16.5-generated neurons. (m1-m2) In L5, none of the BrdU+ cells in either WT or mutant co-expressed Neurofilament-H/Smi32. (n1-n3) Co-labelling of BrdU+ cells with UL marker Cux1 in L6 shows an increased number of retained in the LLs Cux $1^{+}$cells most of which express BrdU (arrows). (n3) Statistical analysis (*, $P<0.05, n=3$ per genotype). (01-03) Double-labelling for BrdU and ROR demonstrates the strongly increased numbers of E16.5-born L4 ROR ${ }^{+}$cells in the mutant compared to WT cortex (arrows). (n3) Statistical analysis $\left({ }^{*}, P<0.05, n=3\right.$ per genotype). All countings of the laminar distribution of $\mathrm{BrdU}^{+}$cells (A1/A2,F1/F2,K1/K2) were performed within frames sized $800 \mu \mathrm{m}$ ( $h$ ) $\times$ $400 \mu \mathrm{m}$ (w) spanning the entire cortical thickness, subdivided into 10 equally-sized bins. BrdU/Ctip2 and BrdU/NF colabaling was evaluated in $200 \mu \mathrm{m}(\mathrm{h}) \times 200 \mu \mathrm{m}(\mathrm{w})$ frames, while BrdU/Tbr1, BrdU/Cux1 and BrdU/ROR co-staining was evaluated within $300 \mu \mathrm{m}$ (h) $\times 100 \mu \mathrm{m}(\mathrm{w})$

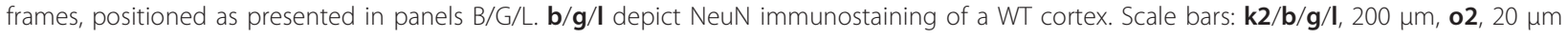

advantage of the fact the pattern of NeuN immunostaining allowed distinguishing the location of LL and $\mathrm{UL}$ in the postnatal cortex (Fig. 2b3-b4), we calculated the percentage of $\mathrm{BrdU}^{+} / \mathrm{NeuN}^{+}$cells located to either LLs (L5-L6) or ULs (L2-L4) out of the total BrdU ${ }^{+} / \mathrm{NeuN}^{+}$cells in frames spanning the entire cortex (Additional file 5: Figure S5). Analysis of the position of the E12.5-born cells revealed no differences in their laminar location between WT and the mutant (Fig. 4a1a3; Additional file 5: Figure S5A1-A3). In contrast, birthdating at E14.5, revealed a significantly larger proportion of $\mathrm{BrdU}^{+}$cells in the deep position of the Zbtb20 lacZ/lacZ cortex, and on contrary, a smaller fraction of such cells was found in the superficial position of the mutants (Additional file 5: Figure S5B1-B3; also Fig. 4f1-f3). Similarly, BrdU pulse labelling at E16.5 (at the peak of UL generation) showed a reduced distribution of tagged cells at superficial position and significantly more deeply located $\mathrm{BrdU}^{+}$cells in the mutant cortex (bins 4-6; Fig. 4k1-k3), that was also evident on BrdU/NeuN double-stained sections (Additional file 5: Figure S5C1-C3).

Using BrdU co-staining with layer-specific neuronal markers, for L5 (Neurofilament/Smi32 and Ctip2) and L6 (Tbr1), we quantitatively investigated the layer fate of cells generated at stages E12.5, E14.5 or E16.5. The results revealed that the mutant Ncx had significantly increased populations of L6 and L5 neurons born at E12.5 (Fig. 4c1-e3), as well as at E14.5, the peak of generation of L4 neurons during normal corticogenesis (Fig. 4h1j2). At E16.5 such expansion of L6/L5 fate identities was no more evident (Fig. 4m1-m2). However, the cells born at E16.5 in the mutant Ncx demonstrated a significant increase of the co-labeling with the L4 marker ROR as compared to the control Ncx (Fig. 4o1-o2, arrows, O3). These results suggest that the developmental window for generation of L6-L5-L4 neurons was expanded by at least 2 days for each neuronal type, which will profoundly affects the progenitor pool size for L3-L2 neurons. In a further support of such a scenario were the results after analysis of the fractions of $\mathrm{Cux}^{+} / \mathrm{ROR}^{+}$ (L4) and $\mathrm{Cux}^{+} / \mathrm{ROR}^{-}$(L3-L2) neuronal subsets born at stages E12.5, E14.5 or E16.5 in WT and mutant mice (Additional file 6: Figure S6). We found that at P12, the UL fractions born at E12.5 or E14.5 did not differ significantly between WT and mutant mice (Additional file 6: Figure S6A1-B3). However, changes were observed in the E16.5-born UL neuronal fractions in Zbtb20 LOF as compared to WT: the L4 fraction was larger, while the L3-L2 fraction was diminished (Additional file 6: Figure $\mathrm{S} 6 \mathrm{C} 1-\mathrm{C} 3)$.

Together, these results strongly suggest that the timed expression of transcriptional repressor $Z b t b 20$ in cortical progenitors (appearing in the entire pallium only after E14.5) could control the transition from early- versus late born neuronal layer identities.

\section{Impaired late neurogenesis and neuronal migration in Zbtb20-deficient cortex}

By using $40 \mathrm{~min}$ BrdU-pulse labelling in vivo at E16.5 (the peak of production of UL neurons) we found a significant reduction of the progenitor proliferation in the 
Zbtb20LOF cortex (Fig. 5a1-a3). Additionally, BrdU/Ki67 double-labelling after a $24 \mathrm{~h} \mathrm{BrdU}$ pulse (E15.5- > E16.5), indicated an increased progenitor exit from the mitotic cycle as measured by the percentage of the $\mathrm{BrdU}^{+} / \mathrm{Ki}^{-} 7^{-}$ cells versus all $\mathrm{BrdU}^{+}$cells (Fig. 5b1-b3). Consistent with these data, we found at E16.5 in the mutant DP a reduction of Tbr2 ${ }^{+}$IPs (Fig. 5c1-c3), the main neuronal source for generation of neurons with an UL neural fate [49]. Notably, we did not detect changes in the progenitor cell exit during early neurogenesis (time window of LL generation) using a $24 \mathrm{~h}$ BrdU pulse at E12.5-> E13.5, followed by BrdU/Ki67 double-labelling (Fig. 5d1-d3).

Our previous expression analysis of Zbtb20 in developing cortex at stage E18.5 suggested a migratory delay of NeuroD $1^{+}$neurons (Fig. $4 \mathrm{~h} 2$ of [35]). At the same stage, we showed here a band of $\mathrm{Id} 2^{+}$, Math $2 / \mathrm{Nex}^{+}$neurons in the pallial SVZ in Zbtb2O KO mice (Additional file 7: Figure S7, arrows), suggesting migratory abnormalities of the lately-born neurons. Indeed, in the UL birthdating experiments at E16.5, the mutant Ncx contained significantly more E16.5-born $\mathrm{BrdU}^{+} / \mathrm{Cux}^{+}$cells in the L6 (Fig. 4n1-n3) as well as in the subcortical white matter (data not shown). Notably, the retained $\mathrm{Cux}^{+}$ cells were $\mathrm{ROR}^{-}$(Fig. 2e2/f2; arrowheads) indicating that a sub-population of correctly specified L3/L2 neurons exhibits an impaired migration towards their final destination to the CP. Notably, the postmitotic expression of Zbtb20 in cortical plate is confined to the $\mathrm{Cux}^{+} / \mathrm{ROR}^{-}$population.
Zbtb20 deficiency affects the expression of CoupTF1 in developing cortex

As noticed, the orphan nuclear receptor CoupTF1, whose strong expression is normally restricted to L4 in the SS area $[45,50]$ was ectopically expressed as a thick band along the entire AP axis of the Zbtb20 mutant (Fig. 6a1-a4; arrows). Similar to the presented here abnormalities of Ncx in Zbtb20 LOF, overexpression of CoupTF1 in the pallial VZ promotes progenitor exit from mitotic cycle, inhibits the IPs production and causes enhanced generation of early-born at the expense of late generated neuronal fates [51]. At E12.5, both CoupTF1 [50, 52] and Zbtb20 are expressed at the corticostriatal border in faint DV gradients. Indeed, the double IHC at E12.5 showed a co-localization of Zbtb20 and CoupTF1 in the VZ of the control animals (Fig. 1c1c3), and significant enhancement of CoupTF1 expression in $Z b t b 20^{\text {lacZ/lacZ }}$ mice as revealed by both ISH (Fig. 6b1-b2) and IHC (Fig. 6C1-C2). Thus, the observed increased generation of early-born neuronal fates (L6,L5) in Zbtb20 LOF might be mediated by modulation of CoupTF1 expression.

To study a possible regulation of CoupTF1 expression by $Z b t b 20$ at molecular level, we investigated whether Zbtb20 binds to the promoter of CoupTF1 [53] (Fig. 6dd2, Additional file 8: Figure S8). In ChIP assays, we used neural stem cultures (NSC) from E15.5 cortices from WT and Zbtb20KO embryos and Zbtb20 antibody. We found that Zbtb20 binds with low affinities with
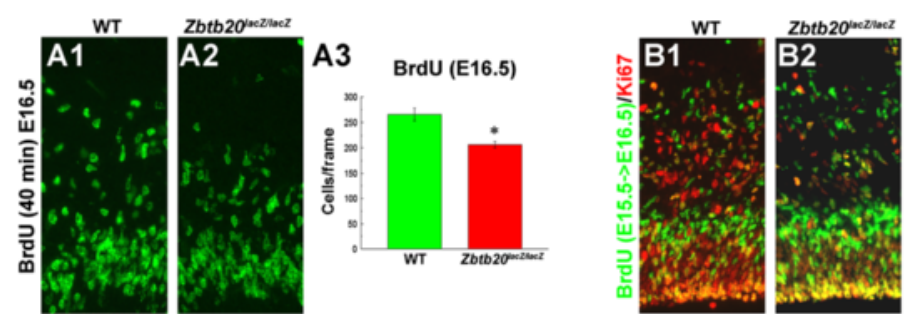

B3 Cell cycle exit (E15.5->E16.5)
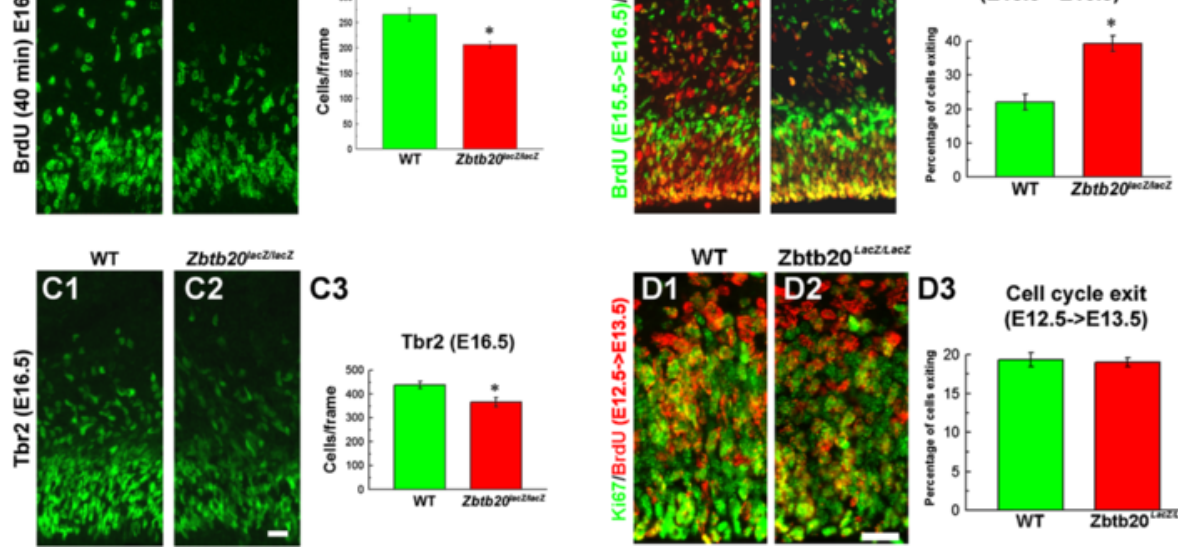

C3
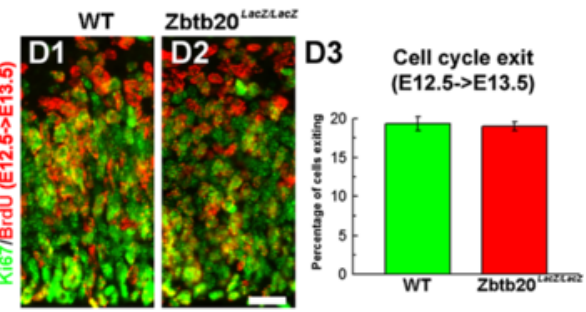

Fig. 5 Altered cell cycle parameters in Zbtb2 laczllacz mice. a1-c3 Impaired cell cycle kinetics in DP of Zbtb20 mutant during late neurogenesis at stage E16.5. a1-a3 Decreased proliferation as measured by short - term (40 min) BrdU pulse labelling. Cells were evaluated in frames sized $300 \mu \mathrm{m}(\mathrm{h}) \times 200 \mu \mathrm{m}(\mathrm{w})$ in DP. b1-b3 Co-immunostaining for BrdU and Ki67 after $24 \mathrm{~h}$ BrdU pulse labelling (E15.5- > E16.5) revealed an increased percentage of cells exiting the cell cycle in the DP of the mutant as compared with the WT cortex. Cells were evaluated in frames sized $300 \mu \mathrm{m}$ (h) $\times 100 \mu \mathrm{m}(w)$ in DP. c1-c3 Labelling with Tbr2 antibody indicates a depletion of intermediate progenitors in Zbtb20 $0^{\text {lac } Z \text { lacz }}$ DP. Cells were evaluated in frames sized $300 \mu \mathrm{m}(\mathrm{h}) \times 200 \mu \mathrm{m}(\mathrm{w})$ in DP. Statistical analysis (a3,b3,c3) proves that the differences are significant $\left({ }^{*}, P<0.05, n=3\right.$ per genotype). d1-d3 Unaltered cell cycle exit kinetics for early (BrdU-tagged at E12.5) progenitors at E13.5. Cells were evaluated in frames sized $150 \mu \mathrm{m}(\mathrm{h}) \times 75 \mu \mathrm{m}$ $(w)$ in DP. No change in the percentage of cells exiting the cell cycle in the mutant DP as calculated by BrdU/Ki67 co-staining after a $24 \mathrm{~h}$ (E12.5- $>$ E13.5) BrdU pulse ( $P>0.05, n=3$ per genotype). Scale bars: $20 \mu m$ 


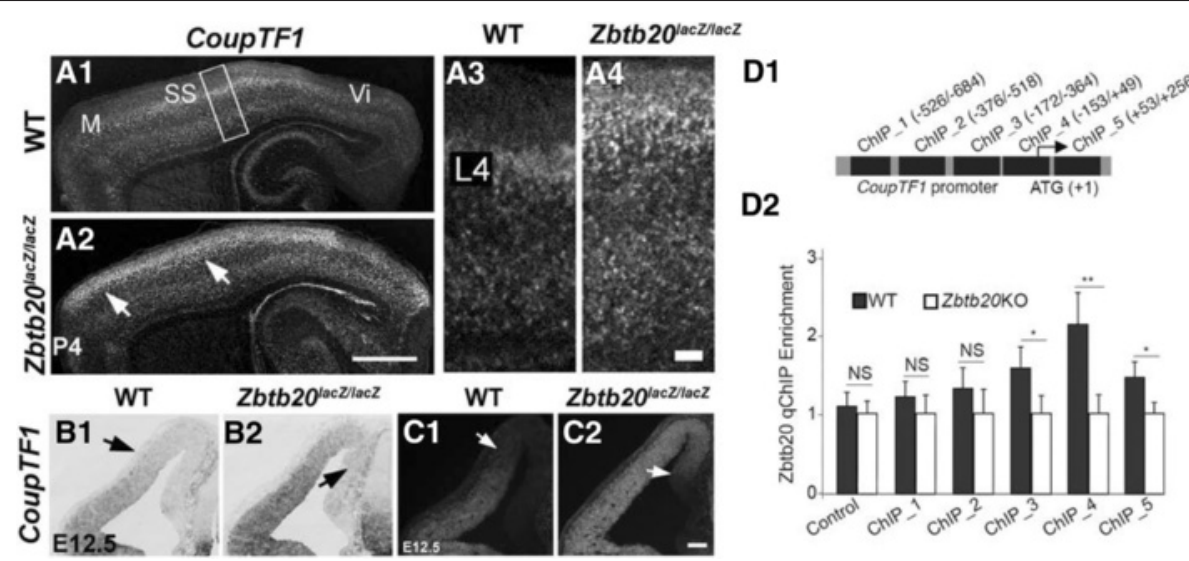

Fig. 6 Regulation of Coup-TF1 expression by Zbtb20. a1-c2 Striking enhancement of the expression of TF CoupTF1 in Zbtb20lacZlacZ mutants. (a1-a4) Ectopic expansion of the CoupTF1 signal in the SS area at P4 as detected by ISH. Note the shift of a strong CoupTF1 signal in a wide band in the ULs of the mutant (arrows in a2). (b1-c2) Ectopic expansion of CoupTF1 expression in mutant DP/MP at stage E12.5, detected either by ISH (b1-b2) or IHC (c1-c2). d1-d2 Zbtb20 binds to the CoupTF1 promoter. d1 The scheme depicts relative positions of fragments on the promoter and the first intron of CoupTF1 that are used in ChIP experiment. d2 ChIP analysis for the CoupTF1 promoter and first intron occupancy by Zbtb20 in cortical neural stem cells from wild type (WT) and Zbtb20 lacz/lacz cortices (as negative control). Scale bars: a2, 1 mm; a4/c2, 100 $\mu \mathrm{m}$

fragments at locations of $-172 /-364$ (ChIP_3) and $+53 /$ +256 (ChIP_5) of the CoupTF1 promoter and the first intron (Additional file 8: Figure S8). Zbtb20 occupies the CoupTF1 promoter with highest affinity at the location of $-153 /+49$ (ChIP_4), which contains multiple DNA binding motifs of Zbtb20 [33] (Fig. 6d1-d2, Additional file 8: Figure S8). These data suggests that TF Z Ztb20 is a regulator of the expression of CoupTF1 most probably acting as a repressor. In a further support, ISH with Zbtb20 in situ probe on E15.5 brain sections from WT and Coup $T F 1^{-/-}$mice [51], showed a decreased mRNA signal (Additional file 9: Figure S9; arrows) suggesting a cross regulatory loop between these two TFs.

\section{Discussion}

Here we identify TF Zbtb20 as essential regulator of the timed sequential generation of distinct neuronal layer identities in developing cortex. Our results suggest that by executing a robust expression in the germinative zone of the entire pallium after E14.5, the Zbtb20 imposes cell-intrinsic temporal limits for generation of L6-L4 versus L3-L2 neuronal fates.

\section{Zbtb20 is a regulator of timed neurogenesis in developing neocortex}

During corticogenesis, the timed generation of layerspecific fates depends on intrinsic and extrinsic cues acting at a given developmental stage [1]. This process includes consecutive steps of repression of cell fates generated during earlier stage. Here we showed that in a lack of TF Zbtb20 in the cortical progenitors, neurons with L6-L5-L4 identity continue to be produced heterochronicly, in an expanded by at least 2 days time window, thus substantially shortening the time for production of L3 and especially of L2 neurons (Fig. 7). We consider that this temporal alteration of the schedule for layer production together with the disclosed enhanced exit from mitosis of E16.5 progenitors are the main factors leading to L6-L5-L4 enlargement in the Zbtb20LOF. Because of its dynamics of expression, Zbtb20 exerts different roles at distinct developmental stages: at E14.5, the time at which the full extent of Zbtb20 expression in the pallial VZ progenitors is achieved, this TF restricts L6-L5 versus L4 fate, while at E16.5, the peak of UL generation, Zbtb20 restricts L4 versus L3-L2 fate. In addition, in Zbtb20 LOF cohorts of progenitors leave the cell cycle prematurely, diminishing the progenitor pool that remains for the latest-born neuronal types.

Genes known to regulate the sequential transitions between cortical subtypes include CoupTF1 [51, 54, 55], FoxG1 [14, 56], Gli3 [57] and Brn2 [58]. The expression of neither FoxG1 [35] nor Gli3 (this study, data not shown) were altered in $Z b t b 20^{\text {lacZ/lacZ }}$ mice. Interestingly, both Zbtb20 and CoupTF1 exerted a similar expression dynamics, starting in progenitors of VP, LP at E12.5 and progressively expanding at later stages in VZ/ SVZ of the entire pallium, and even continue to be colocalized postnatally in UL neurons. As shown here, Zbtb20 binds the promoter of CoupTF1, and in Zbtb2O LOF the expression of CoupTF1 was elevated as early as E12.5, indicating that Zbtb20 controls directly the expression of CoupTF1. Notably, several aspects of the neocortical phenotype in Zbtb20KO mice are reminiscent to abnormalities observed after in vivo overexpression of CoupTF1 [51], including: (i) expansion of early born neuronal sets, (ii) changed balance between early 


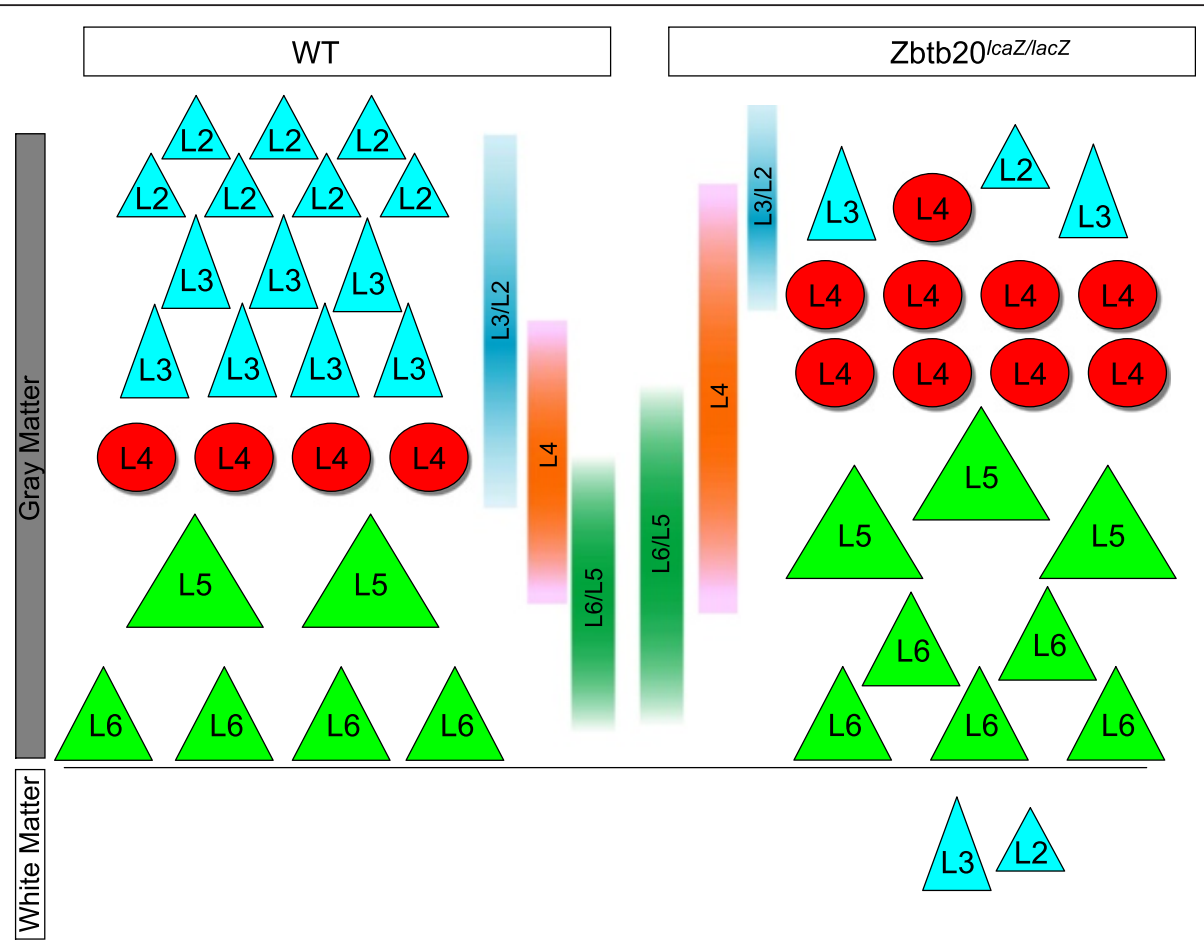

Fig. 7 Schematic summary of the observed effects of Zbtb20 on the temporal acquisition of identity by the cortical glutamatergic projection neurons. a Arrangement of cortical pyramidal neurons in WT mice and their normal time windows of generation. $\mathbf{b}$ Layer defects in Zbtb20 $0^{\text {lacZllacz }}$ mice and the respective changes in the time windows for neuronal generation. Note the increase of $L 6, L 5$ and $L 4$ neurons and the diminishing of $L 3$ and $L 2$ neurons. A few ROR ${ }^{+}(L 4)$ cells can even be seen intermingled with $L 3 / L 2$ cells. Some $L 3 / L 2$ cells are retained in the subcortical white matter and LLS

and late born neurons, (iii) enhanced cell cycle exit of progenitors and (iv) diminished production of IPs. On the contrary, in the pallium of CoupTF1 KO mice, the expression of Zbtb20 was reduced, making possible the existence of a feedback loop between $Z b t b 20$ and CoupTF1. The lack of birthdating and cell cycle abnormalities during L6-L5 neurogenesis in the mutant pallium during the early neurogenesis indicates that Zbtb20 most probably exerts effects on temporal specification of the LLs via the ectopic CoupTF1 expression.

Acting at the border between the archi- and isocortex, Zbtb2O repression seems to be important to inhibit neocortical cell fate [33]. Upon overexpression in progenitors of medial pallium, Zbtb20 directly binds and represses the activity of genes that are specifically expressed in LL neurons (Tbr1, FoxP2, Fezf2, Ctip2, Sox5) or ULs (e.g. Rorb, Satb2, Cux1/Cux2) [33]. However, in developing Ncx, Zbtb20 co-exists with, and thus might directly repress, only few of those TFs: Fezf2 and FoxP2 (in mitotic VZ precursors), Cux1/2 (in mitotic cells in VZ/SVZ as well as in postmitotic cells in ULs), and Satb2/Brn2 (in postmitotic ULs). The enhancement of markers which do not co-exist in the same cells with Zbtb20, such as Ctip2 (L6 and L6) and ROR (L4), can be explained by action of $Z b t b 20$ at the level of the progenitors of these neurons. The expansion of Ctip $2^{+} \mathrm{L} 5$ subsets in Zbtb20 LOF of neurons most probably involves a derepression of the Fezf2 gene in VZ/SVZ. Together with the results in [33], our data suggest that TF Zbtb2O acts as a modulator of the Fezf2/Ctip2/Tbr1/ Satb2 network mostly in pallial VZ progenitors.

The mutant phenotype of the latest-born $\mathrm{Zbtb} 20^{+} \mathrm{L3}-$ L2 cells suggests also an involvement of Zbtb20 at the postmitotic level, probably influencing their proper migration towards the cortical plate. The involvement of Zbtb20 in the regulation of neuronal migration of lateborn UL cells was supported by our findings indicating: (i) retention of $\mathrm{Cux}^{+} / \mathrm{ROR}^{-}$(Fig. 2f2, arrowheads) and Bhlhb5 $^{+}$(Additional file 3: Figure S3 F2, inset) cells below the ULs of the mutant at P12; (ii) their birth date at E16.5 (Fig. 4n2); (iii) accumulation of postmitotic cells in the mutant SVZ at E18.5 (Additional file 7: Figure S7). Thus, the prenatal reduction of UL precursor pool is possibly exacerbated by a postnatal migrational deficit of L3-L2 neurons. Potential target genes for this deficit are TFs Cux1 which is expressed in both mitotic and postmitotic progenitors [19], and Brn2 [40] which was recently shown as a possible Zbtb20 downstream target [59]. 


\section{Conclusions}

Transcription factor $Z b t b 20$ is expressed in the ventricular zone of the developing neocortex in a dynamic spatiotemporal pattern. Loss of Zbtb20 in cortical radial glia cells leads to prolongation of the developmental time limits for sequential production of early-born cortical layer identities (L6, L5 and L4). This dramatically shortens the time of production of later born (L3, L2) neuronal sets which are severely underrepresented. Mechanistically, the deficiency of Zbtb20 leads to decreased proliferation and enhanced exit from mitotic cycle of the late cortical progenitors, an effect mediated at least in part via modulation of the expression of CoupTF1/Nr2f1.

\section{Methods}

\section{Animal experiments}

Animals were handled in accordance with the German Animal Protection Law, after an approval of the experiments by the Niedersächsische Landesamt für Verbraucherschutz und Lebesmittelsicherheit (LAVES)/ Oldenburg, contract No 33.9-42502-04-11/0622 from 07.12.2011. Experiments were completed prior to January $1^{\text {st }}$ 2013. All surgical procedures were performed under isoflurane $/ \mathrm{N}_{2} \mathrm{O}$ anesthesia, and all efforts were made to minimize suffering. The $Z b t b 20$ gene targeting and the generation of the transgenic mice have been previously described [35]. The Zbtb20 knock out (KO) mice lack the functionally important $\mathrm{BTB} / \mathrm{POZ}$ domain of the protein as well as the first of five zinc fingers, which were replaced by a lacZ-neomycin cassette. Therefore, homozygous mutants will be referred to as $Z b t b 20^{\text {lacZ/lacZ }}$ mice within this study. The specificity of the deletion and the complete loss of Zbtb20 protein have been confirmed as described [35].

\section{ChIP analysis}

ChIP analyses were performed as described previously [60] with some modifications. Cortical progenitors were prepared from E15.5 WT and Zbtb20KO mice [35] and cultured as described [61]. ChIP experiments were performed using an EZ ChIP assay kit (Millipore), according to the supplier's instructions with Zbtb20 antibody (HPA016815, Sigma). A genomic fragment of the Trim11 gene and a GFP antibody plus IgG were used as negative DNA and antibody controls, respectively [60]. Primer sequences are listed Additional file 10: Table S1.

\section{Histological processing}

Isolated embryos or brains at defined stages were washed in cold phosphate-buffered saline (PBS) and fixed in $4 \%$ paraformaldehyde (PFA) overnight at $4{ }^{\circ} \mathrm{C}$. Tissues were rinsed in PBS, and processed for standard cryo-embedding. Cryosections (16 $\mu \mathrm{m}$ thick) were washed and blocked for $1 \mathrm{~h}$ in blocking solution containing a normal serum. Primary antibodies were incubated overnight at $4{ }^{\circ} \mathrm{C}$ in the blocking solution. After washing, the sections were incubated with speciesspecific secondary antibodies from the Alexa series (Invitrogen) in blocking solution for $2 \mathrm{~h}$ at room temperature (RT), washed again and mounted with Vectashield mounting-medium (Vector Labs) containing DAPI. We used the following primary antibodies/dilutions: mouse anti- $\beta$-galactosidase (1:200; Promega, Madison, WI), rat anti-BrdU (1:200; Abcam), mouse anti-BrdU (1:50; BD Bioscience), goat anti-Brn2 (1:50, Santa Cruz Biotech, Santa Cruz, CA), rabbit anti-Caspase-3 (1:200; Cell Signalling, Cambridge, UK), mouse anti-CoupTF1 (1:1000; Perseus Proteomics, Tokyo, Japan), rat anti-Ctip2 (1:200; Abcam), rabbit anti-Cux1 (1:250; Santa Cruz), rabbit anti-FoxP1 (1:500, Abcam), rabbit anti-FoxP2 (1:500, Abcam), rat anti-Ki67 (1:100; Dako), mouse anti-Nestin (1:100; Millipore, Billerica, MA), mouse anti-NeuN (1:100; Millipore), mouse anti-Neurofilament (1:100; Abcam), rabbit anti-Pax6 (1:300; Covance), mouse antiPax6 (1:100; Developmental Studies Hybridoma Bank, DHSB), mouse anti-phospho-Histone H3 (1:50; Cell Signalling), mouse anti-phospho-Vimentin (1:300; MBL), mouse anti-ROR (1:100; Perseus Proteomics), mouse anti-Satb2 (1:200; Abcam), mouse anti-Sox2 (1:50; R\&D systems), rabbit anti-Tbr1 (1:300; Abcam), rabbit antiTbr2 (1:200; Chemicon), rabbit anti-Zbtb20 (1:25-1:100, Sigma). The anti-BrdU antibodies were visualized after pre-treatment of tissues in $2 \mathrm{~N} \mathrm{HCl}$ at $37{ }^{\circ} \mathrm{C}$ for $30 \mathrm{~min}$. The anti-Zbtb20 antibody was used after an antigen retrieval by heating in a microwave $(800 \mathrm{~W}, 3$ times, 5 min each) in a citrate buffer ( $\mathrm{pH} 6.0$ ).

\section{In situ hybridization}

Whole heads from E12.5 or whole brains from E18.5 or $\mathrm{P} 4$ mice were dissected in ice-cold DEPC-treated PBS, fixed in $4 \%$ PFA/PBS for $3 \mathrm{~h}$ at $4{ }^{\circ} \mathrm{C}$, washed in PBS, and incubated in $25 \%$ sucrose overnight at $4{ }^{\circ} \mathrm{C}$. Specimens were sectioned at $16 \mu \mathrm{m}$ after embedding and freezing in OCT cryomatrix (Leica Microsystems Nussloch GmbH, Wetzlar, Germany). Nonradioactive in situ hybridization was done as described [27].

\section{Image analysis and quantification}

Images were captured with an Olympus BX60 microscope, a Leica DM6000 epifluorescent system or a laser confocal microscope (Leica Sp5). For cell counts in sections from wild type (WT) and homozygous brains, we blindly counted the positive cells within equally sized frames (size of frames provided in the figure legends) on coded cross sections of somatosensory cortex in WT and mutant mice ( $\mathrm{n} \geq 3$ per genotype). For BrdU birthdating experiments, frames spanning the entire cortex on cross 
brain sections were divided into 10 equally-sized bins, the $\mathrm{BrdU}^{+}$cells in every bin were counted and divided by the total number of $\mathrm{BrdU}^{+}$cells in all 10 bins. The size of the counting frames for BrdU/marker colocalization are provided in the figure legends.

Laser confocal microscopy was used to verify colocalization of multiple fluorescent signals. We performed $\mathrm{Z}$ sectioning at $0.5-1 \mu \mathrm{m}$ intervals and optical stacks of at least 10 images were used for analysis, by the Leica Advanced Fluorescence software version 2.3.6. All images were processed with Adobe Photoshop (Version CS2) by overlaying the pictures, adjusting brightness, contrast and size.

\section{Statistical analysis}

Statistical evaluation was performed by Student's $T$-test or one-way ANOVA followed by Tukey-Kramer's post hoc analysis. Statistical significance between control and experimental condition was considered if $p<0.05$. Data are presented as means \pm s.e.m.

\section{Additional files}

Additional file 1: Figure S1. Expression of TF Zbtb20 in developing pallium. (A) B-gal staining of Zbtb20lacZ+/- embryos at E11.5. The arrow points to a ß-gal activity expanding from the subpallium into the $V Z$ of LP, while DP remains negative. (B) ISH on sagittal E14.5 brain sections reveals Zbtb20 expression in the entire pallial VZ (arrows). Expression in $\mathrm{MZ}$ is depicted by arrowheads. (C1-C2) ISH on cross E16.5 brain sections demonstrates a strong Zbtb20 expression in the pallial VZ (asterisk), gradually decreasing in the SVZ (C2). Also evident is a positive signal in the lateral migratory stream (arrow) as well as in $\mathrm{MZ}$ (arrowheads). The image in C2 corresponds to the boxed area in C1. BG, basal ganglia; DG, dentate gyrus, DP, dorsal pallium; Hi, hippocampus; LP, lateral pallium; Ncx, neocortex; Str, striatum; MP, medial pallium, VP, ventral pallium. Scale bars: A, $100 \mu \mathrm{m} ; \mathrm{B} / \mathrm{C} 1500 \mu \mathrm{m} ; \mathrm{C} 2,100 \mu \mathrm{m}$. (PDF 451 kb)

Additional file 2: Figure S2. Expression of TF ZBTB20 in UL neurons in early postnatal stage (P4) neocortex. (A1-A3) Double immunostaining for Zbtb20 and Brn2, and an overlay on brain cross section. Arrows depict double-positive cells at uppermost position in the developing Ncx. (B1) Immunostaining for TF Ctip2 (counterstained for DAPI) on brain cross section marks L5. The frame corresponds to the position of images shown in B2-B4. (B2-B4) Few L5 neurons express Zbtb20. Co-staining for Zbtb20 and Ctip2, and an overlay on brain cross sections. Arrows depict Zbtb20 ${ }^{+}$cells in $L 5$ which do not exhibit co-labeling for Ctip2. (C1) Immunostaining for nuclear receptor ROR (counterstained for DAPI) on brain cross section (the strong ROR immunosignal marks L4). The frame corresponds to the position of images shown in C2-C4. (C2-C4) Co-staining for Zbtb20 and ROR, and an overlay on brain cross sections. The $\mathrm{Zbtb} 2 \mathrm{O}^{+}$neurons in the upper layers do not exhibit co-labeling for ROR (arrows). Scale bars: A3, $50 \mu \mathrm{m} ; \mathrm{B} 1 / \mathrm{C} 1,100 \mu \mathrm{m} ; \mathrm{B} 4 / \mathrm{C} 4,20 \mu \mathrm{m}$. (PDF $161 \mathrm{~kb}$ )

Additional file 3: Figure S3. Cortical arealization in Zbtb20lacZ/lacZ mice. (A1-C4) ISH analysis on brain sagittal sections at matched levels revealing enhancement of L5 neuronal subsets, marked by Fezf2 (A1-A4), Clim 1 (B1-B4) and Id2 (C1-C4), in the Zbtb20 lacz/lacz mutants. The pattern of Id2 expression confirms the increase of both L6 and L5 neurons, accompanied by a drastic decrease of the ULs in the Zbtb20KO cortex at P4. Asterisk in C1/C2 points to the presumptive border between the motor (M) and somatosensory (SS) area which appears rostrally displaced in the mutant. Images in A3/A4, B3/B4 and C3/C4 correspond to a field in SS cortex (indicated by a frame in $A 1, B 1, C 1)$. (D1-F2) However, ISH staining for Cadherin 8 (D1-D2) and IHC labelling for Serotonin (E1-E2) and Bhlhb5 (F1-F2) on sagittal sections indicates a normal position of the M/SS border (asterisk). Scale bar: C2, 1 mm. (PDF 176 kb)

Additional file 4: Figure S4. Enhanced presence of lower layer neurons in primary motor cortex of Zbtb20 lacZ/lacZ mutants. (A1-A2) NeuN IHC demonstrating an overview of the motor cortex (depicted on the scheme in the upper left corner) on cross brain sections. The enlargement of $L 6$ and $L 5$ and the thinning of L2-L4 is apparent. (B1-B3) Decreased numbers of L2-L4 Cux1-immunostained neurons in Zbtb20-deficient cortex. (C1-D3) Immunostaining with FoxP1 $(C 1, C 2)$ and Ctip2 $(D 1, D 2)$ antibodies revealed in the Zbtb20 mutant an increased number of both FoxP2 ${ }^{+}$L6 and Ctip2 ${ }^{+}$L5 neurons. (B3,C3,D3) Graphs representing statistical evaluation of the results ( ${ }^{*}, P<0.05, n=3$ per genotype). All stainings were performed at stage $P 8$. Countings were performed within frames sized $400 \mu \mathrm{m}(\mathrm{h}) \times 300 \mu \mathrm{m}(\mathrm{w})$ for FoxP2, $300 \mu \mathrm{m}(\mathrm{h}) \times 300 \mu \mathrm{m}(\mathrm{w})$ for Ctip2, $500 \mu \mathrm{m}(\mathrm{h}) \times 300 \mu \mathrm{m}$ (w) for Cux1. Scale bars: A2, $200 \mu \mathrm{m}, \mathrm{B} 2 /$ C2/D2, $100 \mu \mathrm{m}$. (PDF $183 \mathrm{~kb}$ )

Additional file 5: Figure S5. Distribution of neurons born at E12.5, E14.5 and E16.5 in WT and Zbtb20lacZ/lacZ mice across neocortical layers. BrdU was injected at each of the above pointed embryonic stages and the neocortical layers were visualized by NeuN IHC. We then calculated the percentage of BrdU ${ }^{+} / \mathrm{NeuN}^{+}$cells in L6, L5 and L2-L4 out of the total $\mathrm{BrdU}^{+} / \mathrm{NeuN}^{+}$cells in a frame $800 \mu \mathrm{m}(\mathrm{h}) \times 200 \mu \mathrm{m}(\mathrm{W})$ spanning through L2-L6. The image on the left side of the figure depicts NeuN immunostaining of a WT cortex (identical with Fig. 4b,g,l) providing an overview of the cortical layers. (A1-A3) BrdU (E12.5- > P8)/NeuN double $\mathrm{IHC}$ demonstrates the predominant distribution of $\mathrm{BrdU}^{+}$cells in the deep (L5-L6) layers. No significant differences between the WT and mutant mice were detected (A3, $P>0.05, n=3$ per genotype). (B1-B3) BrdU (E14.5- > P12)/NeuN double IHC depicts a larger proportion doublepositive cells in deep ( $\mathrm{L} 5$-L6) layers of the mutant cortex (arrows in B2), while a lower percentage in the superficial (L2-L4) layers (B3, ${ }^{*}, P<0.05$, $n=3$ per genotype). (C1-C3) BrdU (E16.5-> P12)/NeuN double IHC reveals a larger proportion double-positive cells in mutant deep (L5-L6) layers (arrows in C2), while a lower percentage in the superficial (L2-L4) layers ( $C 3,{ }^{*}, P<0.05, n=3$ per genotype). LL, lower neocortical layers; $U L$, upper neocortical layers. Scale bar: C2, $50 \mu \mathrm{m}$. (PDF $139 \mathrm{~kb}$ )

Additional file 6: Figure S6. Birth date analysis of specific subpopulations of neurons within the superficial (L2-L4) neocortical layers of WT and Zbtb20 lacZllacZ mice. BrdU was injected at E12.5, E14.5 and E16.5 and BrdU IHC was combined with Cux1 and ROR immunostaining to distinguish between $\mathrm{L} 4\left(\mathrm{Cux} 1^{+} / \mathrm{ROR}^{+}\right)$and $\mathrm{L} 2-\mathrm{L} 3\left(\mathrm{Cux} 1^{+} / \mathrm{ROR}^{-}\right)$neuronal subsets. The $\mathrm{BrdU}^{+} / \mathrm{Cux} 1$ ${ }^{+} / \mathrm{ROR}^{+}$and the $\mathrm{BrdU}^{+} / \mathrm{Cux} 1^{+} / \mathrm{ROR}^{-}$neuronal subsets were calculated as a percentage of the BrdU ${ }^{+}$cells within frames located in the upper layers within SS cortex. The same animals and corresponding frames were used as for Fig. 4 and Additional file 5: Figure S5. (A1-A3) Analysis of UL phenotypes born at stage E12.5. BrdU ${ }^{+} / \mathrm{Cux} 1^{+} / \mathrm{ROR}^{+}$cells are depicted by arrows, while the $\mathrm{BrdU}^{+} / \mathrm{CuX}^{+} / \mathrm{ROR}^{-}$cells - by arrowheads. No significant differences in the proportions of $\mathrm{Cux} 1^{+} / \mathrm{ROR}^{+}(\mathrm{L} 4)$ and $\mathrm{Cux}^{+} / \mathrm{ROR}^{-}(\mathrm{L} 2-\mathrm{L} 3)$ cells, born at this stage, were observed between the $W T$ and the mutants $(A 3, P>0.05, n$ $=3$ per genotype). (B1-B3) Analysis of UL phenotypes born at stage E14.5. $\mathrm{BrdU}^{+} / \mathrm{Cux} 1^{+} / \mathrm{ROR}^{+}$cells are depicted by arrows, while the $\mathrm{BrdU}^{+} / \mathrm{Cux} 1^{+} / \mathrm{ROR}$ cells - by arrowheads. Similarly to stage E12.5, no significant differences in the proportions of $\mathrm{Cux} 1^{+} / \mathrm{ROR}^{+}$and $\mathrm{Cux}^{+} / \mathrm{ROR}^{-}$cells, born at E14.5, were observed between the WT and the mutants (A3, $P>0.05, n=3$ per genotype). (C1-C3) Analysis of UL phenotypes born at stage E16.5. $\mathrm{BrdU}^{+} / \mathrm{Cu} \times 1^{+} / \mathrm{ROR}^{+}$cells are depicted by arrows, while the $\mathrm{BrdU}^{+} / \mathrm{Cux} 1^{+} / \mathrm{ROR}^{-}$ cells - by arrowheads. Note that a significantly larger proportion of $\mathrm{Cux} 1^{+} / \mathrm{ROR}$ ${ }^{+}(\mathrm{L} 4)$ cells are born at stage E16.5 in the mutant ULs than in the WT ULS (C3, ${ }^{*}, P<0.05, n=3$ per genotype). At the same time, in the Zbtb20 deficient cortex, a significantly smaller percentage of BrdU ${ }^{+}$cells showed L2-L3 (Cux1 $\left.{ }^{+} / \mathrm{ROR}^{-}\right)$identity $\left(\mathrm{C} 3,{ }^{*}, P<0.05, n=3\right.$ per genotype). Countings were performed within frames sized $300 \mu \mathrm{m}(\mathrm{h}) \times 100 \mu \mathrm{m}(\mathrm{w})$ spanning L2-L4. Scale bar: C2, $20 \mu \mathrm{m}$. (PDF $135 \mathrm{~kb}$ )

Additional file 7: Figure S7. Retention of postmitotic cells in ectopic positions in Zbtb20 mutant cortex at E18.5. RNA ISH for TFs Id2 (A1-A2), Math2) NEX (B1-B2), and NeuroD1 (C1-D2) in DP (A1-C2) and pyriform cortex (D1-D2) of WT and mutant mice. Ectopic location of cells positive for the three TFs in the mutant is depicted by arrows. Scale bar: $200 \mu \mathrm{m}$. (PDF $1980 \mathrm{~kb}$ ) 
Additional file 8: Figure S8. Map of the CoupTF1 promoter and binding sites of Zbtb20. The DNA sequence of the CoupTF1 promoter (Salas et al. [53]) contains multiple DNA binding motifs (motif_1, motif_2, motif_3) of Zbtb20 [33]. Note that Zbtb20 binds to the CoupTF1 promoter with highest affinity at the DNA fragment ChIP 4 (as depicted in Fig. 6d1-d2), covering these motifs. (PDF 147 kb)

Additional file 9: Figure S9. Expression of Zbtb20 in CoupTF ${ }^{-/-}$mice. ISH analysis at stage E15.5 using Zbtb20 in situ probe on cross sections from WT (A1) and CoupTF1 ${ }^{-1-}$ (A2) embryo brains. Note the marked reduction of the $\mathrm{Zbtb20} \mathrm{ISH}$ signal in VP of CoupTF ${ }^{-/-}$mutants (arrows in A2). Scale bar: $200 \mu \mathrm{m}$. (PDF $427 \mathrm{~kb}$ )

Additional file 10: Table S1. Primer sequences used in the study. (PDF $35 \mathrm{~kb}$ )

\section{Abbreviations}

$\mathrm{CP}$, cortical plate; CSMN, corticospinal motor neurons; DP, dorsal pallium; GOF, gain-of-function; Hi, hippocampus; LL, lower layer; LOF, loss-of-function; LP, lateral pallium; MZ, marginal zone; Ncx, neocortex; pVim, phosphorylated vimentin; RGCs, radial glial cells; SS, somatosensory; SVZ, subventricular zone; TCA, thalamocortical axons; TF, transcription factor; UL, upper layer; VP, ventral pallium; $\mathrm{VZ}$, ventricular zone

\section{Acknowledgements}

We are grateful to M. Daniel and S. Schlott for outstanding technical assistance. We thank the following researchers for providing us with in situ probes: E.A. Grove, D.D.M. O'Leary, V. Tarabykin, F. Guillemot, M. Hibi, M. Lie, J. Liu, M. Price, T. Rabbitts, V. Tarabykin, S. Tole, and J. Rubenstein.

\section{Funding}

This work was supported by the Max Planck Society (AS), The DFG * Center for Nanoscale Microscopy and Molecular Physiology of the Brain (CNMPB) (AS, TCT), and the Alexander von Humboldt Foundation (ABT).

\section{Authors' contributions}

$A B T$ and $A S$ designed research, ABT and TCT performed research, EHR contributed to generating Zbtb20KO mice; AS and ABT analyzed data; MS provided embryo brains from CoupTF1KO mutant, AS and ABT wrote the manuscript. All authors read and approved the final manuscript.

\section{Competing interests}

The authors declare that they have no competing interests.

\section{Consent for publication}

Not applicable.

\section{Ethics approval and consent to participate}

No experiments were done with human beings. Animal experiments have been approved by the Ethics Committee of Lower Saxony.

\section{Author details}

'Molecular Developmental Neurobiology Laboratory, Max Planck Institute of Biophysical Chemistry, Am Fassberg, 37077 Gottingen, Germany. ${ }^{2}$ Center for Nanoscale Microscopy and Molecular Physiology of the Brain (CNMPB), 37075 Göttingen, Germany. ${ }^{3}$ Department of Anatomy, Histology and Embryology, Medical University-Varna, Varna, Bulgaria. ${ }^{4}$ Molecular Neurobiology Group, Institute of Neuroanatomy, University of Goettingen Medical Center, Goettingen, Germany. ${ }^{5}$ University Nice Sophia Antipolis, iBV UMR 7277, F-06108 Nice, France. ${ }^{6}$ Inserm, iBV, U1091, F-06108 Nice, France.

Received: 11 January 2016 Accepted: 21 May 2016 Published online: 09 June 2016

\section{References}

1. McConnell SK, Kaznowski CE. Cell cycle dependence of laminar determination in developing neocortex. Science. 1991;254:282-5.

2. Angevine Jr JB, Sidman RL. Autoradiographic study of cell migration during histogenesis of cerebral cortex in the mouse. Nature. 1961;192:766-8.

3. Takahashi T, Nowakowski RS, Caviness Jr VS. The mathematics of neocortical neuronogenesis. Dev Neurosci. 1997;19:17-22.
4. Nowakowski RS, Caviness Jr VS, Takahashi T, Hayes NL. Population dynamics during cell proliferation and neuronogenesis in the developing murine neocortex. Results Probl Cell Differ. 2002;39:1-25.

5. Rakic P. Specification of cerebral cortical areas. Science. 1988;241:170-6.

6. Kwan KY, Sestan N, Anton ES. Transcriptional co-regulation of neuronal migration and laminar identity in the neocortex. Development. 2012;139: $1535-46$.

7. Alcamo EA, Chirivella L, Dautzenberg M, Dobreva G, Fariñas I, Grosschedl R, et al. Satb2 regulates callosal projection neuron identity in the developing cerebral cortex. Neuron. 2008;57:364-77.

8. Britanova O, de Juan Romero C, Cheung A, Kwan KY, Schwark M, Gyorgy A, et al. Satb2 is a postmitotic determinant for upper-layer neuron specification in the neocortex. Neuron. 2008;57:378-92.

9. Hevner RF, Shi L, Justice N, Hsueh Y, Sheng M, Smiga S, et al. Tbr1 regulates differentiation of the preplate and layer 6. Neuron. 2001;29:353-66.

10. Joshi PS, Molyneaux BJ, Feng L, Xie X, Macklis JD, Gan L. Bhlhb5 regulates the postmitotic acquisition of area identities in layers $I I-\mathrm{V}$ of the developing neocortex. Neuron. 2008;60:258-72.

11. Kwan KY, Lam MM, Krsnik Z, Kawasawa YI, Lefebvre V, Sestan N. SOX5 postmitotically regulates migration, postmigratory differentiation, and projections of subplate and deep-layer neocortical neurons. Proc Natl Acad Sci U S A. 2008;105:16021-6.

12. McKenna WL, Betancourt J, Larkin KA, Abrams B, Guo C, Rubenstein JL, et al. Tbr1 and Fezf2 regulate alternate corticofugal neuronal identities during neocortical development. J Neurosci. 2011;31:549-64.

13. Srinivasan K, Leone DP, Bateson RK, Dobreva G, Kohwi Y, Kohwi-Shigematsu $T$, et al. A network of genetic repression and derepression specifies projection fates in the developing neocortex. Proc Natl Acad Sci U S A. 2012;109:19071-8.

14. Hanashima C, Li SC, Shen L, Lai E, Fishell G. Foxg1 suppresses early cortical cell fate. Science. 2004;303:56-9.

15. Arlotta P, Molyneaux BJ, Chen J, Inoue J, Kominami R, Macklis JD. Neuronal subtype-specific genes that control corticospinal motor neuron development in vivo. Neuron. 2005;45:207-21.

16. Frantz GD, Weimann JM, Levin ME, McConnell SK. Otx1 and Otx2 define layers and regions in developing cerebral cortex and cerebellum. J Neurosci. 1994;14:5725-40.

17. Tarabykin V, Stoykova A, Usman N, Gruss P. Cortical upper layer neurons derive from the subventricular zone as indicated by Svet1 gene expression. Development. 2001;128:1983-93.

18. Zimmer C, Tiveron MC, Bodmer R, Cremer H. Dynamics of Cux2 expression suggests that an early pool of SVZ precursors is fated to become upper cortical layer neurons. Cereb Cortex. 2004;14:1408-20.

19. Nieto M, Monuki ES, Tang H, Imitola J, Haubst N, Khoury SJ, et al. Expression of Cux-1 and Cux-2 in the subventricular zone and upper layers II-IV of the cerebral cortex. J Comp Neurol. 2004;479:168-80.

20. Franco SJ, Gil-Sanz C, Martinez-Garay I, Espinosa A, Harkins-Perry SR, Ramos $C$, et al. Fate-restricted neural progenitors in the mammalian cerebral cortex. Science. 2012;337:746-9.

21. Seuntjens E, Nityanandam A, Miquelajauregui A, Debruyn J, Stryjewska A, Goebbels S, et al. Sip1 regulates sequential fate decisions by feedback signaling from postmitotic neurons to progenitors. Nat Neurosci. 2009;12: 1373-80.

22. Elsen GE, Hodge RD, Bedogni F, Daza RA, Nelson BR, Shiba N, et al. The protomap is propagated to cortical plate neurons through an Eomesdependent intermediate map. Proc Natl Acad Sci U S A. 2013;110:4081-6.

23. Mallamaci A, Stoykova A. Gene networks controlling early cerebral cortex arealization. Eur J Neurosci. 2006;23:847-56.

24. O'Leary DD, Sahara S. Genetic regulation of arealization of the neocortex. Curr Opin Neurobiol. 2008;18:90-100

25. Sur M, Rubenstein JL. Patterning and plasticity of the cerebral cortex. Science. 2005;310:805-10.

26. Zembrzycki A, Chou SJ, Ashery-Padan R, Stoykova A, O'Leary DD. Sensory cortex limits cortical maps and drives top-down plasticity in thalamocortical circuits. Nat Neurosci. 2013;16:1060-7.

27. Muhlfriedel S, Kirsch F, Gruss P, Chowdhury K, Stoykova A. Novel genes differentially expressed in cortical regions during late neurogenesis. Eur J Neurosci. 2007;26:33-50

28. Zhang W, Mi J, Li N, Sui L, Wan T, Zhang J, et al. Identification and characterization of DPZF, a novel human BTB/POZ zinc finger protein sharing homology to BCL-6. Biochem Biophys Res Commun. 2001;282:1067-73. 
29. Mitchelmore C, Kjaerulff KM, Pedersen HC, Nielsen JV, Rasmussen TE, Fisker $M F$, et al. Characterization of two novel nuclear BTB/POZ domain zinc finger isoforms. Association with differentiation of hippocampal neurons, cerebellar granule cells, and macroglia. J Biol Chem. 2002;277:7598-609.

30. Kelly KF, Daniel JM. POZ for effect-POZ-ZF transcription factors in cancer and development. Trends Cell Biol. 2006;16:578-87.

31. Nielsen JV, Nielsen FH, Ismail R, Noraberg J, Jensen NA. Hippocampus-like corticoneurogenesis induced by two isoforms of the BTB-zinc finger gene Zbtb20 in mice. Development. 2007;134:1133-40.

32. Nielsen JV, Blom JB, Noraberg J, Jensen NA. Zbtb20-induced CA1 pyramidal neuron development and area enlargement in the cerebral midline cortex of mice. Cereb Cortex. 2010;20:1904-14.

33. Nielsen JV, Thomassen M, Møllgård K, Noraberg J, Jensen NA. Zbtb20 defines a hippocampal neuronal identity through direct repression of genes that control projection neuron development in the isocortex. Cereb Cortex. 2014;24:1216-29.

34. Xie Z, Ma X, Ji W, Zhou G, Lu Y, Xiang Z, et al. Zbtb20 is essential for the specification of CA1 field identity in the developing hippocampus. Proc Natl Acad Sci U S A. 2010;107:6510-5.

35. Rosenthal EH, Tonchev AB, Stoykova A, Chowdhury K. Regulation of archicortical arealization by the transcription factor Zbtb20. Hippocampus. 2012;22:2144-56.

36. Vuillaume ML, Delrue MA, Naudion S, Toutain J, Fergelot P, Arveiler B, et al, Expanding the clinical phenotype at the $3 q 13.31$ locus with a new case of microdeletion and first characterization of the reciprocal duplication. Mol Genet Metab. 2013;110:90-7.

37. Cordeddu V, Redeker B, Stellacci E, Jongejan A, Fragale A, Bradley TE, et al. Mutations in ZBTB20 cause Primrose syndrome. Nat Genet. 2014;46:815-7.

38. Rasmussen MB, Nielsen JV, Lourenco CM, Melo JB, Halgren C, Geraldi CV, et al. Neurodevelopmental disorders associated with dosage imbalance of ZBTB20 correlate with the morbidity spectrum of ZBTB20 candidate target genes. J Med Genet. 2014;51:605-13.

39. Lowther C, Costain G, Melvin R, Stavropoulos DJ, Lionel AC, Marshall CR, et al. Adult expression of a 3q13.31 microdeletion. Mol Cytogenet. 2014;7:23.

40. McEvilly RJ, de Diaz MO, Schonemann MD, Hooshmand F, Rosenfeld MG. Transcriptional regulation of cortical neuron migration by POU domain factors. Science. 2002;295:1528-32.

41. Sugitani $Y$, Nakai S, Minowa O, Nishi M, Jishage K, Kawano H, et al. Brn-1 and Brn-2 share crucial roles in the production and positioning of mouse neocortical neurons. Genes Dev. 2002;16:1760-5.

42. Ino H. Immunohistochemical characterization of the orphan nuclear receptor ROR alpha in the mouse nervous system. J Histochem Cytochem. 2004;52:311-23.

43. Nakagawa $Y, O^{\prime}$ Leary DD. Dynamic patterned expression of orphan nuclear receptor genes RORalpha and RORbeta in developing mouse forebrain. Dev Neurosci. 2003;25:234-44.

44. Hisaoka T, Nakamura Y, Senba E, Morikawa Y. The forkhead transcription factors, Foxp1 and Foxp2, identify different subpopulations of projection neurons in the mouse cerebral cortex. Neuroscience. 2010;166:551-63.

45. Zhou C, Tsai SY, Tsai MJ. COUP-TFI: an intrinsic factor for early regionalization of the neocortex. Genes Dev. 2001;15:2054-9.

46. Bulchand S, Subramanian L, Tole S. Dynamic spatiotemporal expression of LIM genes and cofactors in the embryonic and postnatal cerebral cortex. Dev Dyn. 2003;226:460-9.

47. Neuman T, Keen A, Zuber MX, Kristjansson Gl, Gruss P, Nornes HO. Neuronal expression of regulatory helix-loop-helix factor ld2 gene in mouse. Dev Biol. 1993;160:186-95.

48. Rubenstein JL, Anderson S, Shi L, Miyashita-Lin E, Bulfone A, Hevner R. Genetic control of cortical regionalization and connectivity. Cereb Cortex. 1999;9:524-32

49. Gotz M, Huttner WB. The cell biology of neurogenesis. Nat Rev Mol Cell Biol. 2005:6:777-88

50. Liu Q, Dwyer ND, O'Leary DD. Differential expression of COUP-TFI, CHL1, and two novel genes in developing neocortex identified by differential display PCR. J Neurosci. 2000;20:7682-90.

51. Faedo A, Tomassy GS, Ruan Y, Teichmann H, Krauss S, Pleasure SJ, et al. COUP-TFI coordinates cortical patterning, neurogenesis, and laminar fate and modulates MAPKJERK, AKT, and beta-catenin signaling. Cereb Cortex. 2008;18:2117-31.

52. Qiu Y, Cooney AJ, Kuratani S, DeMayo FJ, Tsai SY, Tsai MJ. Spatiotemporal expression patterns of chicken ovalbumin upstream promoter-transcription factors in the developing mouse central nervous system: evidence for a role in segmental patterning of the diencephalon. Proc Natl Acad Sci U S A. 1994;91:4451-5.

53. Salas R, Petit FG, Pipaon C, Tsai MJ, Tsai SY. Induction of chicken ovalbumin upstream promoter-transcription factor I (COUP-TFI) gene expression is mediated by ETS factor binding sites. Eur J Biochem. 2002;269:317-25.

54. Naka H, Nakamura S, Shimazaki T, Okano H. Requirement for COUP-TFI and II in the temporal specification of neural stem cells in CNS development. Nat Neurosci. 2008;11:1014-23.

55. Tomassy GS, De Leonibus E, Jabaudon D, Lodato S, Alfano C, Mele A, et al. Area-specific temporal control of corticospinal motor neuron differentiation by COUP-TFI. Proc Natl Acad Sci U S A. 2010;107:3576-81.

56. Kumamoto T, Toma K, Gunadi, McKenna WL, Kasukawa T, Katzman S, et al. Foxg1 coordinates the switch from nonradially to radially migrating glutamatergic subtypes in the neocortex through spatiotemporal repression. Cell Rep. 2013;3:931-45.

57. Wang H, Ge G, Uchida $Y$, Luu B, Ahn S. Gli3 is required for maintenance and fate specification of cortical progenitors. J Neurosci. 2011;31:6440-8.

58. Dominguez MH, Ayoub AE, Rakic P. POU-III transcription factors (Brn1, Brn2, and Oct6) influence neurogenesis, molecular identity, and migratory destination of upper-layer cells of the cerebral cortex. Cereb Cortex. 2013;23: 2632-43.

59. Nagao M, Ogata T, Sawada Y, Gotoh Y. Zbtb20 promotes astrocytogenesis during neocortical development. Nat Commun. 2016;7:11102.

60. Tuoc TC, Stoykova A. Trim11 modulates the function of neurogenic transcription factor Pax6 through ubiquitin-proteosome system. Genes Dev. 2008;22:1972-86.

61. Conti L, Pollard SM, Gorba T, Reitano E, Toselli M, Biella G, et al. Nicheindependent symmetrical self-renewal of a mammalian tissue stem cell. PLoS Biol. 2005;3(9):e283.

\section{Submit your next manuscript to BioMed Central and we will help you at every step:}

- We accept pre-submission inquiries

- Our selector tool helps you to find the most relevant journal

- We provide round the clock customer support

- Convenient online submission

- Thorough peer review

- Inclusion in PubMed and all major indexing services

- Maximum visibility for your research

Submit your manuscript at www.biomedcentral.com/submit
C Biomed Central 